\title{
Multiple Coherent Components in Heavy-Ion Collisions
}

\author{
E. Ikonen ${ }^{* \dagger}$ \\ Metrology Research Institute, Aalto University and Centre for Metrology and Accreditation, \\ P.O. Box 13000, FI-00076 Aalto, Finland \\ E-mail: erkki.ikonenetkk.fi
}

\begin{abstract}
Multiple coherent source components for particle correlation experiments are considered with partially coherent pion radiation produced by heavy-ion collisions. If the partial coherence in pion radiation will ever be conclusively demonstrated, the source properties producing this radiation become under study. This work contributes to the understanding of properties of such particle radiation. The conventional analysis, which assumes one coherent source current, is extended here to cover the case of multiple coherent source currents. Theoretical calculations on the relation between two- and three-pion correlators give some evidence, when compared with experimental data, for the existence of multiple coherent components in heavy-ion collisions.
\end{abstract}

XXth Hadron Collider Physics Symposium

November 16 - 20, 2009

Evian, France

\footnotetext{
* Speaker.

${ }^{\dagger}$ This study was supported by the Academy of Finland under grant no. 129971.
} 


\section{Introduction}

The existence of partial coherence in nuclear collisions has been discussed for over forty years $[1,2]$. In principle, the effect could be demonstrated by the zero-momentum-difference intercept of the two-pion correlation function. However, such an experimental observation of partial coherence is demanding because of the effects due to long-lived resonances, particle misidentification, and final state interactions, making extrapolation of the finite-momentum data to the zero-difference limit a very challenging task. A partial solution to the above problem is provided by measurement of genuine correlations of three pions and scaling of the result by the genuine two-pion correlation function with exponent 3/2 [3]. Long-lived resonances and particle misidentification then influence in the same way both the numerator and denumerator. The normalized three-particle correlator $r_{3}$ obtained by such calculation offers, in principle, an unbiased estimate of partial coherence: an experimental value of $r_{3} / 2$ smaller than one would provide the desired signature of partial coherence in pion radiation.

In fact, there already exist measurement results from which values $r_{3} / 2<1$ have been extrapolated to the vanishing momentum difference [4, 5, 6, 7], but the extrapolation method used in those references has been questioned [8]. Thus there is not yet generally accepted evidence of partial coherence in heavy-ion collisions. However, such evidence might be obtained by threepion correlations and proper analysis of either existing or future experimental data, which would make the source properties of the partially coherent pion radiation an interesting research topic. There is no a priori reason why only one coherent source component should exist as assumed in Refs. $[2,3,7]$. On the contrary, results on photon radiation from free-electron lasers suggest the existence of multiple coherent components $[9,10,11,12]$. Extension of the theory with one coherent source component to multiple coherent source components in heavy-ion collisions appears not to have been presented before Ref. [13]. Finally, it is noted that tentative application of the developed theory, assuming that the extrapolation biases of Ref. [8] are negligible, suggests existence of multiple coherent source components in partially coherent pion radiation.

\section{Normalized two- and three-pion correlators}

In analogy with photon correlations, probabilities $P_{1}\left(p_{1}\right), P_{2}\left(p_{1}, p_{2}\right)$, and $P_{3}\left(p_{1}, p_{2}, p_{3}\right)$ describe emission of one, two, and three pions or photons with momenta $p_{i}(i=1,2,3)[2,10,14]$. Their explicit form is calculated in Ref. [13] for the case of multiple coherent source components. The zero-momentum-difference intercept of the two-pion correlation function is obtained as the two-pion correlator $R_{2}(p, p)=\left[P_{2}(p, p)-P_{1}(p)^{2}\right] / P_{1}(p)^{2}$, where the contribution of random single-pion events $P_{1}(p)^{2}$ is removed from $R_{2}(p, p)$ to account only for the genuine two-pion effects. Similarly the normalized three-pion correlator is given by $r_{3}=R_{3}(p, p, p) /\left[R_{2}(p, p)\right]^{3 / 2}$, where the contributions by single- and two-pion events are removed in $R_{3}(p, p, p)$ [3]. For a fixed value of the number $N$ of coherent processes [13]

$$
r_{3} / 2=\left\{3 R_{2}(p, p)-2+2\left[1-R_{2}(p, p)\right]^{3 / 2} / \sqrt{N}\right\} /\left[R_{2}(p, p)\right]^{3 / 2}
$$

gives the quantitative relation between the normalized three-pion correlator and the two-pion correlator, as shown in Fig.1. 


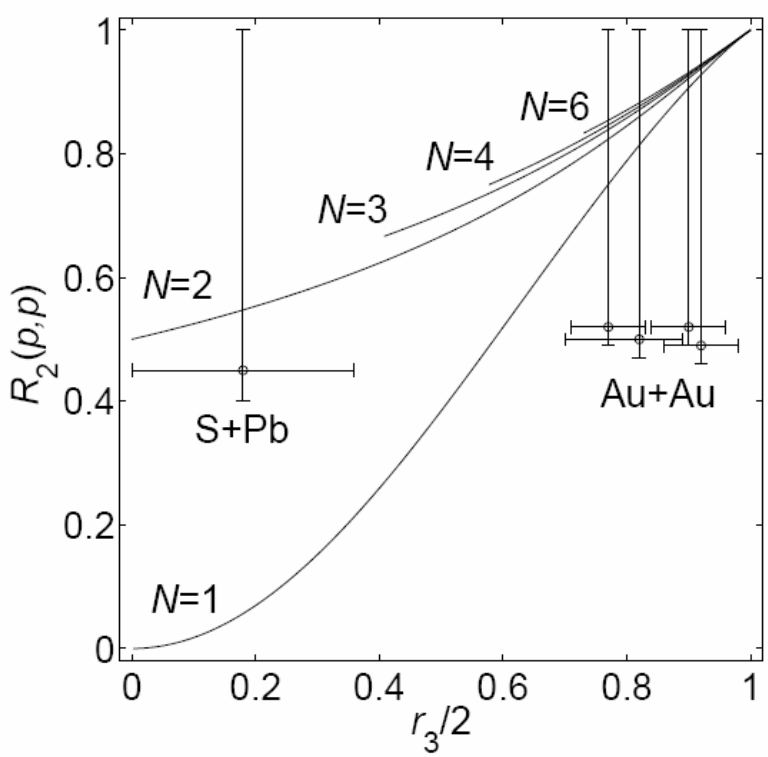

Fig. 1. Relation between $r_{3} / 2$ and $R_{2}(p, p)$ for different numbers $N$ of coherent source components and data from $\mathrm{S}+\mathrm{Pb}$ [4] and $\mathrm{Au}+\mathrm{Au}$ [7] collisions. Parameter $R_{2}(p, p)$ is the zero-momentumdifference intercept of the two-pion correlation function. In the normalized three-pion correlator $r_{3} / 2$, the effects of long-lived resonances, particle misidentification, and experimental binning are cancelled [3]. The result with a single coherent component, used in the analysis by Adams et al. [7], is shown by the curve labeled $N=1$. Experimental data from $\mathrm{S}+\mathrm{Pb}$ collisions are in agreement with the curve $N=2$ [13].

\section{References}

[1] G. N. Fowler and R. M. Weiner, Phys. Lett. B 70, 201 (1977).

[2] M. Gyulassy, S. K. Kauffmann, and L. W. Wilson, Phys. Rev. C 20, 2267 (1979).

[3] U. Heinz and Q. H. Zhang, Phys. Rev. C 56, 426 (1997).

[4] H. Bøggild et al., Phys. Lett. B 455, 77 (1999).

[5] M. M. Aggarwal et al., Phys. Rev. Lett. 85, 2895 (2000).

[6] I. G. Bearden et al., Phys. Lett. B 517, 25 (2001).

[7] J. Adams et al., Phys. Rev. Lett. 91, 262301 (2003).

[8] U. Heinz and A. Sugarbaker, Phys. Rev. C 70, 054908 (2004).

[9] R. Bonifacio, L. De Salvo, P. Pierini, N. Piovella, and C. Pellegrini, Phys. Rev. Lett. 73, 70 (1994).

[10] E. Ikonen, J. Opt. Soc. Am. B 21, 1403 (2004).

[11] V. Ayvazyan et al., Phys. Rev. Lett. 88, 104802 (2002).

[12] T. Shintake et al., Nature Photon. 2, 555 (2008).

[13] E. Ikonen, Phys. Rev. C 78, 051901(R) (2008); 80, 019903 (E) (2009).

[14] E. Ikonen and S. Holopainen, Phys. Rev. A 76, 031801(R) (2007). 